\title{
Routinen der Gründungsförderung in der Grundsicherung
}

Die Gründungsförderung der Arbeits- und Sozialverwaltung hat im vergangenen Jahrzehnt deutliche Spuren im allgemeinen Gründungsgeschehen in Deutschland hinterlassen. Die Fördermodalitäten und -zahlen der einzelnen Instrumente (Ich-AG, Überbrückungsgeld, Einstiegsgeld, Gründungszuschuss) unterscheiden sich dabei erheblich voneinander sowie im Zeitverlauf. In diesem Beitrag blicken wir am Beispiel des Einstiegsgeldes in der Grundsicherung (Sozialgesetzbuch Zweites Buch (SGB II)) hinter die Kulissen der Jobcenter und zeigen, in welchem Spannungsfeld von widersprüchlichen Erwartungen sich die Integrationsfachkräfte bewegen. Gründungen unter den Bedingungen von Hartz IV zu begleiten, bedeutet auch für die Jobcenter eine ungewöhnliche Herausforderung.

STEFAN BERNHARD, HANS J. PONGRATZ

\section{Einleitung}

Die Gründungsförderung der Arbeits- und Sozialverwaltung kann auf eine langjährige, wechselvolle Geschichte zurückblicken (Caliendo/Kritikos 2007; Caliendo et al. 2009). Lange vor den Hartz-Reformen wurden das Überbrückungsgeld ( $\$ 55 \mathrm{a}$ Arbeitsförderungsgesetz und $\$ 57$ Sozialgesetzbuch Drittes Buch (SGB III)) und die Gründungsförderung im Bundessozialhilfegesetz (BSHG) ( $\$ 18$ Abs. 5) eingeführt. Mit den Hartz-Reformen (Deutscher Bundestag 2003) hat die Gründungsförderung neue Aufmerksamkeit auf sich gezogen und ist zum Gegenstand wiederholter Reformen geworden. Insbesondere im SGB III zeigt sich der Gesetzgeber reformfreudig: Der begleitend zum Überbrückungsgeld im Jahr 2003 eingeführte Existenzgründungszuschuss („Ich-AG“, $₫ 421$ Abs. 1 SGB III) wurde im August 2006 mit dem Überbrückungsgeld in den Gründungszuschuss überführt ( $\$ 57$ SGB III). Dieser wiederum wurde fünf Jahre später grundlegend umgestaltet und u. a. von einer Pflicht- in eine Ermessensleistung umgeformt (nun $\$ \$ 93$ und 94 SGB III). Im Vergleich dazu weisen die gesetzlichen Regelungen zur Gründungsförderung im SGB II eine größere Konstanz auf. Die Vorschriften zum Einstiegsgeld sind seit ihrer Einführung im Jahr 2005 unverändert, allerdings lassen sich geschäftspolitische Neubewertungen des Instruments in den Jobcentern beobachten.
Dabei zeichnet sich gerade die Lage der Leistungsbeziehenden im SGB II zumindest in finanzieller Hinsicht durch einen Mangel an Ressourcen aus, der wenig Spielraum für unternehmerische Aktivitäten bietet. Die Jobcenter stehen vor der Frage: Unter welchen Bedingungen kann ein in vielfacher Hinsicht voraussetzungsvolles Gründungsprojekt (Finanzierung, Fähigkeiten, Marktzugang etc.) von Personen erfolgreich geplant und umgesetzt werden, die sich gerade mit Erfahrungen beruflichen Scheiterns und/oder sozialer Hilfebedürftigkeit konfrontiert sehen? Demgegenüber sind die Kompetenzen der Fachkräfte im Jobcenter ganz auf den Arbeitsmarkt ausgerichtet; die Beurteilung der wirtschaftlichen Aussichten einer Existenzgründung stellt für sie eine ebenso ungewohnte wie ungewöhnliche Arbeitsaufgabe dar. Die Absicherung des Risikos der Arbeitslosigkeit war im deutschen Sozialsystem lange ausschließlich auf abhängige Beschäftigung ausgerichtet - ebenso wie die Maßnahmen zu ihrer Überwindung (vgl. den Beitrag von Bothfeld/Rosenthal in diesem Heft). Mit den eingangs genannten Instrumenten der Gründungsförderung wurde Selbstständigkeit als alternative Integrationsoption in Erwerbsarbeit prinzipiell anerkannt, aber in der gesetzlichen und verwaltungstechnischen Umsetzung in unterschiedlicher Weise zugänglich gemacht.

Die Unentschlossenheit von Gesetzgeber und Verwaltung in der Bewertung der Existenzgründung als Erwerbsalternative findet deutlichen Niederschlag in den Förderzahlen (Abbildung 1). Im SGB III erlebt die Förderung nach 
Einführung der Ich-AG einen einmaligen Höhepunkt mit über 350.000 Förderungen im Jahr 2004 (einschließlich Überbrückungsgeld). Nach Zusammenführung der Instrumente im Gründungszuschuss sinken die Zahlen zunächst, bleiben aber auf einem beträchtlichen Niveau zwischen ca. 120.000 bis 145.000 Eintritten in den Jahren von 2007 bis 2011. Zu dieser Zeit ist der Gründungszuschuss der teuerste Einzelposten der aktiven Arbeitsmarktpolitik im SGB III und liegt bei etwa $30 \%$ aller Aufwendungen. Nach der jüngsten Reform zum Jahreswechsel 2011/2012 bricht die Förderung massiv ein und liegt seither unter 2.000 Zugängen pro Monat. Auch beim Einstiegsgeld lässt sich ein früher Höhepunkt bei der Förderung beobachten, allerdings insgesamt auf einem deutlich niedrigeren Niveau. In den Jahren 2006 und 2007 liegt die Zahl der Förderungen noch bei über 30.000 pro Jahr, bevor sie bis 2012 kontinuierlich auf nur noch 6.200 Förderungen zurückgeht. Diese Schwankungen hinterlassen ihre Spuren im allgemeinen Gründungsgeschehen in Deutschland. Dem KfW-Gründungsmonitor 2013 zufolge hat sich der Anteil der Gründer aus Arbeitslosigkeit (einschließlich nicht geförderter Gründungen) auf etwa $10 \%$ im Jahr 2012 verringert, nachdem er in den Vorjahren auf bis zu $20 \%$ angestiegen war. Die Autoren führen das neben der guten Arbeitsmarktlage explizit auch auf die Neugestaltung des Gründungszuschusses zurück (Metzger/Ullrich 2013, S. 9; vgl. auch Sternberg et al. 2013).

In diesem Beitrag richten wir den Blick ins Innere der Arbeits- und Sozialverwaltung, die mit der Aufgabe der Gründungsförderung betraut ist. Der Fokus liegt auf dem Einstiegsgeld im SGB II, wobei sich grundlegende Überlegungen und wichtige Beobachtungen auch auf die Gründungsförderung im SGB III übertragen lassen. Wir stellen Ergebnisse einer Implementationsstudie des Einstiegsgelds vor, die im Jahr 2011 vom Institut für Arbeitsmarkt- und Berufsforschung (IAB) Nürnberg in Zusammenarbeit mit dem Institut für Sozialwissenschaftliche Forschung (ISF) München durchgeführt worden ist (Pongratz et al. 2013a; Bernhard et al. 2013). Das gesetzliche Ziel der Einstiegsgeldförderung ist die - zumindest perspektivische - Überwindung der Hilfebedürftigkeit der Gründenden ( $\$ 16 \mathrm{c}$ Abs. 1 SGB II). Der Auftrag besteht darin, zur Gründung geeignete Personen mit tragfähigen Gründungsprojekten auszuwählen. Wie diese Auswahl unter den spezifischen Bedingungen der Jobcenter konkret zu bewältigen ist, unterliegt recht allgemein gehaltenen Bestimmungen.

In den Jobcentern bestehen deshalb unterschiedliche und teils widersprüchliche Orientierungsmöglichkeiten und -notwendigkeiten (Abschnitt 2): Sie sind an die Steuerungslogik der manageriellen Bürokratie der Bundesagentur für Arbeit (BA) angeschlossen (Standardisierung), sie haben die besonderen Bedingungen des Einzelfalls zu berücksichtigen (Einzelfallorientierung) und sie müssen nicht zuletzt die Erfolgsaussichten eines Gründungsvorhabens bewerten (Gründungsberatung). Diese Erwartungen sind in die gesetzlichen und organisationalen Vorgaben eingeschrieben und sollen von den Integrationsfachkräften berücksichtigt
ABB. 1

\section{Förderung von Existenzgründungen durch Arbeitsagenturen und Jobcenter 2003 - 2012*}

Angaben in absoluten Zahlen

Ich-AG Überbrückungsgeld Gründungszuschuss Einstiegsgeld

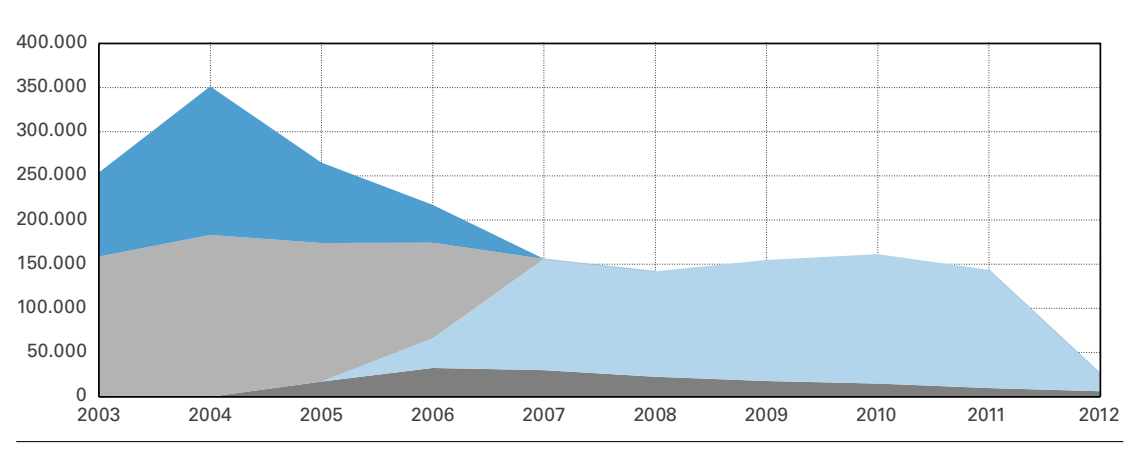

*Gesamtes Bundesgebiet inklusive Optionskommunen und gemeinsameTrägerschaften.

Quelle: Statistiken der Bundesagentur für Arbeit (prozessproduzierte Daten); Berechnungen der Autoren.

werden. Auf der Grundlage einer multiperspektivischen Implementationsstudie (3) stellen wir die These auf, dass in dieser Situation in den Jobcentern Bearbeitungsroutinen ausgebildet werden, um wenigstens einen Teil der institutionalisierten Erwartungen zu bedienen und so eine prekäre Balance zwischen ihnen zu halten (4). Abschließend wenden wir uns der Frage zu, welche Konsequenzen aus den Beobachtungen für die Gründungsförderung in der Arbeitsund Sozialverwaltung zu ziehen sind (5).

\section{Erwartungen an die Gründungs- förderung der Grundsicherung}

\subsection{Leistungen}

Die Verbindung von Grundsicherung für Arbeitsuchende und Gründungsförderung zum Aufbau einer selbstständigen Existenz, wie sie das Instrument des Einstiegsgeldes leisten soll, stellt eine komplexe und widerspruchsvolle Anforderung dar. Im Unterschied zur langjährigen Regelung des Gründungszuschusses (bis Ende 2011) wird das Einstiegsgeld als Ermessensleistung vergeben, wobei sich das Ermessen sowohl auf das Ob der Förderung (EntschlieBungsermessen) als auch auf das Wie der Förderung (Dauer und Höhe, Auswahlermessen) erstreckt. Rechtlich ist eine Förderdauer von bis zu zwei Jahren möglich, empirisch läuft der überwiegende Teil der Förderungen nicht länger als ein halbes Jahr (Pongratz et al. 2013a, S. 21). Die Ermessensausübung hinsichtlich der Förderhöhe ist mittlerweile in 
einer Einstiegsgeld-Verordnung geregelt, die das Bundesministerium für Arbeit und Soziales auf Grundlage von $₫ 16$ Abs. 3 SGB II zum 1. August 2009 erlassen hat. In den meisten Fällen wird von einer Grundförderung von $50 \%$ des maßgebenden Regelsatzes ausgegangen, bei einem allein lebenden Single also von $191 €$, und dieser Betrag kann dann in Abhängigkeit von der Dauer der vorangegangenen Arbeitslosigkeit und der Größe der betreffenden Bedarfsgemeinschaft erhöht werden. In keinem Fall aber soll das Einstiegsgeld über dem vollen Regelsatz der Grundsicherung (derzeit $382 €$ ) liegen. Parallel dazu können zur Deckung des Lebensunterhalts weiter - aufstockend zu dem in der Selbstständigkeit erzielten Gewinn - Leistungen der Grundsicherung bezogen werden. Dem im Vergleich zur durchschnittlichen Förderhöhe des Gründungszuschusses niedrigen Niveau steht die gesetzlich formulierte Erwartung gegenüber, dass sowohl die gründende Person geeignet als auch das Gründungsprojekt wirtschaftlich tragfähig sein soll. Die Regelungen zum Einstiegsgeld (Ermessensentscheidung, Prüfung von Gründungsprojekt und Gründerperson) spiegeln damit einerseits die hohen Anforderungen an eine Selbstständigkeit wider, andererseits verweisen sie auf den institutionellen Kontext des Grundsicherungsauftrags des SGB II: Es soll vermieden werden, durch eine im Verhältnis zum Grundsicherungsniveau hohe Förderhöhe Anreize zur leichtfertigen Aufnahme einer selbstständigen Tätigkeit zu setzen.

\subsection{Erwartungshorizonte der Gründungs- förderung}

Bei der Untersuchung der Gründungsförderung im SGB III konnten institutionalisierte Erwartungen identifiziert werden, die sich analytisch auf drei Erwartungshorizonte mit je eigenen Logiken rückbeziehen lassen (Bernhard 2012a; 2012b). Die Erwartungshorizonte der Einzelfallorientierung, der Gründungsberatung und der Standardisierung sind unabhängig von den konkreten Fördermodalitäten und werden von uns deshalb im Folgenden auch auf die Analyse des Einstiegsgeldes angewendet.

(1) Der Erwartungshorizont der Einzelfallorientierung zielt auf die Berücksichtigung der individuellen Gesamtsituation der Betroffenen ab. Klienten suchen die Verwaltung aufgrund einer spezifischen Problemlage oder konkreten Leistungserwartung auf und versuchen, ihr individuelles Interesse wirkungsvoll zur Geltung zu bringen. Die Auswahl und Umsetzung angemessener Verwaltungsmaßnahmen erfordert die Kenntnis und die Berücksichtigung dieser Besonderheiten des Einzelfalls. Die Dienstleistungsforschung hat den Fluchtpunkt dieses Erwartungshorizontes formuliert (Dunkel 2011): Es geht darum, Dienstleistungen als von „den nachfragenden Subjekten als Ko-Produzenten ausgehenden und mitgesteuerten professionellen Handlungsmodus“ (Hielscher/Ochs 2012, S. 252) zu organisieren. Die Gültigkeit dieser Erwartungen wurde durch die Ein- führung einer Beratungskonzeption in den Jahren $2009 \mathrm{im}$ SGB III bzw. 2013 im SGB II unterstrichen (BA 2009, 2013). Demnach sollen u.a. ein „positives Gesprächsklima“ hergestellt, dann (aufeinander aufbauend) eine Situationsanalyse vorgenommen, Ziele definiert und Lösungsstrategien entwickelt werden. Die Ziele sollen "gemeinsam“ gefunden, die Lösungen zudem „kundenspezifisch“ gestaltet und die „Kunden“ abschließend zur Zielerreichung motiviert werden.

(2) Im Erwartungshorizont der Gründungsberatung (Bernhard 2012b) entstehen Anforderungen an die Jobcenter, wie sie aus verschiedenen Formen der Beratung von Gründenden und Selbstständigen zur Verbesserung der wirtschaftlichen Aussichten bekannt sind. Eine typische Aufgabe bildet die Prüfung und Optimierung eines Businessplans, was die Beurteilung der Eignung des Gründers und der Marktchancen des Projektes einschließt. Bei bereits Selbstständigen müssen Geschäftsunterlagen ausgewertet werden. Wie im Intranet der Bundesagentur spezifiziert ist, bezieht sich das u.a. auf die Prüfung von Geschäftsprozessen, Rechnungswesen, Produkt- und Dienstleistungsangebot, Marketingstrategie, Chancen- und Risikoanalyse, Finanzbedarfsplan und Rentabilitätsvorschau. Die Jobcenter sollen bei dieser Aufgabe durch Externe auf dem Wege einer Einschätzung zur Tragfähigkeit oder über begleitende Maßnahmen unterstützt werden. Offen bleibt dabei, wie die Aussichten einer Selbstständigkeit mit den verfügbaren Alternativen am Arbeitsmarkt ins Verhältnis zu setzen sind.

(3) Der Erwartungshorizont der bürokratischen Standardisierung ist zunächst an der Sicherstellung des staatlichen Grundsatzes der Gleichbehandlung und an der Effizienz der Verwaltungsarbeit ausgerichtet. Im Sinne von Webers Bürokratietheorie werden Präzision und Berechenbarkeit der organisatorischen Abläufe angestrebt. Im Zuge der Hartz-Reformen wurde dieses Modell substanziell in Richtung einer manageriellen Bürokratie umgeformt, bei der wirkungsorientierte Zielsteuerung zur klassischen Regelsteuerung hinzugetreten ist (Schütz 2009). Maßgeblich ist in diesem Zusammenhang das sogenannte Vier-PhasenModell (4PM) mit begleitenden Standardisierungs- und Kontrollverfahren. Mit dem Modell sollen Integrationsfachkräfte Einzelfälle kategorisieren und entsprechende Integrationsziele festlegen - ein notwendigerweise paradoxes Unterfangen, gerade mit Blick auf die Regulative des Dienstleistungsversprechens. Denn die Zuordnung zu den vorgegebenen Kategorisierungen und den dafür vorgesehenen Handlungsstrategien beraubt den Einzelfall gerade all jener Spezifika, die ihn zu einem solchen machen. Ist die Kategorisierung erst einmal erfolgt, schränkt sie die Möglichkeiten einer weitergehenden Einzelfallorientierung ein.

Diese institutionalisierten Erwartungen an die Umsetzung der gesetzlichen Regelungen zum Einstiegsgeld in tägliche Praxis in den Jobcentern sind also nicht nur komplex, sondern es gibt auch unmittelbare Widersprüche zwischen ih- 
nen. So steht etwa der Anspruch einer vertrauensbasierten, ergebnisoffenen, kundengelenkten Kommunikation in Konflikt mit den Kontroll- und Sanktionserfordernissen der Sozialverwaltung (und führt zu einem Vertrauensdilemma). Auch ist unklar, inwieweit die Entscheidung über eine Gründungsförderung unabhängig von innerorganisatorischen Steuerungsanreizen und Zielen der Mittelbewirtschaftung geschehen kann. Andere Spannungsfelder werden erst sichtbar, wenn man sich den Zeitdruck und die enge Staffelung der Beratungstermine, die Zahl der Fälle pro Fachkraft, die Heterogenität der Bedürfnislagen und Erwartungen der Klienten sowie die Varianz der beruflichen Vorbildung der Mitarbeiter vor Augen hält. Dabei erfordert der Einzelfallbezug beim Einstiegsgeld, dass die Anspruchsberechtigung auf Leistungsbezug geprüft, die Vermittelbarkeit am Arbeitsmarkt geklärt und die Erfolgsaussichten des Gründungsprojekts beurteilt werden. Unter den restriktiven Bedingungen des Verwaltungsbetriebs ist eine individualisierende Betrachtung aller relevanten Faktoren äußerst schwierig (Individualisierungsdilemma). Wir zeigen im empirischen Teil, welche Umsetzungsroutinen sich in den Jobcentern beim Einstiegsgeld ausgebildet haben und wo sie sich im Schnittfeld der Anforderungen bewegen.

\section{Empirisches Vorgehen}

Die Grundlage unserer Ergebnisse bildet eine Implementationsstudie (Pongratz et al. 2013a; Bernhard et al. 2013), die multiperspektivisch vorgeht und mit Methodentriangulation arbeitet (Flick 2004). Der Forschungsgegenstand der Administration des Einstiegsgeldes in den Jobcentern wird aus drei Richtungen heraus erkundet: aus der Pers- pektive der hierarchischen Steuerungsversuche über Gesetze und organisationale Gestaltungsansprüche in BA und Jobcentern, aus der Perspektive der Auswahl- und Vergabeprozesse durch Integrationsfachkräfte in den Jobcentern bzw. in deren Interaktionen mit Klienten sowie aus dem Jobcenter-externen Blickwinkel der lokalen Kooperationspartner (darunter die fachkundigen Stellen). Die Fallauswahl erfolgte in einem mehrstufigen Verfahren. In einem ersten Schritt errechneten wir auf Basis quantitativer Daten der Statistik der Bundesagentur für Arbeit monatsdurchschnittliche Zugangsraten in die Einstiegsgeldförderung in allen Jobcentern. Auf dieser Datengrundlage zogen wir zwei Dimensionen für die Auswahl der zu untersuchenden Jobcenter heran: die Höhe der relativen Förderaktivität und die Typisierung regionaler Arbeitsmärkte (Rüb/Werner 2007). So wurden 25 Jobcenter als unter quantitativen Aspekten interessante Varianzfälle identifiziert; 22 von ihnen befragten wir in kurzen, telefonischen Leitfadeninterviews zu ihrer Förderpraxis und -strategie ${ }^{1}$ und wählten daraufhin sechs interessante Fälle für eingehende Fallstudien aus (darunter eine Optionskommune).

Bei der Perspektivierung des Forschungsgegenstandes kamen jeweils verschiedene Erhebungsmethoden zum Einsatz (Übersicht 1). Zur Erfassung der organisationalen Entscheidungssteuerung dienten Experteninterviews mit Führungskräften in den Jobcentern sowie Dokumentenrecherchen. Erhoben wurden manifeste und latente

1 Anlaufstelle derTelefoninterviews waren Geschäftsleiter der Jobcenter (oder ihre Stellvertreter), mit denen fünf Interviews geführt wurden. In den übrigen Fällen wurden Interviewpartner aus den Ebenen der Bereichsleitung, der Teamleitung und der Sachbearbeitung benannt.

ÜBERSICHT 1

Anlage der Studie zur Gründungsförderung

\begin{tabular}{l|l|c|l}
\hline Annäherung über & Erhebungsmethode & $\begin{array}{c}\text { Erhebungs- } \\
\text { umfang }\end{array}$ & Datenmaterial für die Auswertung \\
\hline $\begin{array}{l}\text { Organisation, } \\
\text { Leitungen Jobcenter }\end{array}$ & $\begin{array}{l}\text { telefonische Vorerhebung } \\
\text { leitfadengestützte Experteninter- } \\
\text { views, Dokumentenrecherchen }\end{array}$ & 82 & leitfadengestützte Gesprächsprotokolle \\
\hline Vergabeprozess & $\begin{array}{l}\text { problemzentrierte Interviews } \\
\text { teilnehmende Beobachtungen, } \\
\text { Kurzinterviews mit Fachkräften } \\
\text { und Leistungsberechtigten }\end{array}$ & 16 & $\begin{array}{l}\text { Transkriptionen der Audiodateien, Dokumente (z. B. } \\
\text { ermessenslenkende Weisungen), Interviewprotokolle }\end{array}$ \\
\hline & $\begin{array}{l}\text { leitfadengestützte Experteninter- } \\
\text { views, Dokumentenrecherche }\end{array}$ & 7 & $\begin{array}{l}\text { Transkriptionen der Audiodateien, Dokumente (z. B. inter- } \\
\text { ne Prüflisten zur Gründerauswahl), Interviewprotokolle }\end{array}$ \\
\hline externe Akteure & $\begin{array}{l}\text { Transkriptionen der Audiodateien aus Beobachtungen } \\
\text { und Nachbefragungen, Interaktionsfallstudien }\end{array}$ \\
\hline
\end{tabular}


Vorstrukturierungen von Beratungs- und Entscheidungsprozessen in zentralen und lokalen organisationalen Vorgaben. Die Perspektive des Vergabeprozesses erfassten wir erstens über problemzentrierte Interviews mit Integrations- und Leistungssachbearbeitungsfachkräften (Witzel/Reiter 2012), in denen die praktischen Vollzüge im Arbeitsalltag der Einstiegsgeldvergabe im Vordergrund standen. Zweitens wurden Gespräche mit Klienten teilnehmend beobachtet und begleitend dazu Kurzinterviews mit den Integrationsfachkräften und den erwerbsfähigen Leistungsberechtigten geführt. Dieses Datenmaterial fügten wir zu Interaktionsfallstudien zusammen und werteten es über theoriehaltig-ethnographisches Schreiben aus (Hirschauer 2001). Die Kooperationspartner der Jobcenter befragten wir schließlich in Experteninterviews zu ihren Erfahrungen mit der Einstiegsgeldförderung, mit den erwerbsfähigen Leistungsberechtigten sowie mit den Jobcentern. Insgesamt herrschte bei den Jobcentern, deren Mitarbeitern sowie den Kooperationspartnern eine durchweg hohe Teilnahmebereitschaft. Probleme beim Feldzugang gab es lediglich bei den Interaktionsbeobachtungen, da die ohnehin seltenen gründungsbezogenen Termine mit Klienten häufiger ausfielen.

\section{Verfahrensroutinen der Einstiegs- geldvergabe}

Die widersprüchlichen Erwartungshorizonte der Gründungsberatung, der Einzelfallorientierung und der Standardisierung, so zeigen unsere empirischen Ergebnisse, werden durch Prozesse der Routinisierung von Verfahrensweisen bewältigt. Diese sind teils formal geregelt, teils haben sie sich aber auch aufgrund von Erfahrungen und informellen Abstimmungsprozessen in der Bearbeitungspraxis etabliert. Im Folgenden stellen wir vier dieser Routinen vor, die von zentraler Bedeutung für die Einstiegsgeldvergabe sind, und diskutieren, inwieweit sie welchem der Erwartungshorizonte zuneigen.

\subsection{Prozeduralisierung der Auswahlverfahren und Mechanismen der Selbstselektion}

Die Begleitung der Gründungsvorhaben in den Jobcentern ist organisatorisch als eine Stufenfolge angelegt, bei der jede weitere Stufe die Gründenden mit neuen Aufgaben konfrontiert und damit vor die Frage stellt, ob sie den neuerlichen Aufwand auf sich nehmen und ihr Gründungsprojekt weiter verfolgen wollen. Dieser Auswahlmechanismus funktioniert primär in Form von Selbstselektion, die durch von außen (Jobcenter und externe beratende Stellen) herangetragene Anforderungen ausgelöst wird. Diese Stufen sind in den untersuchten Jobcentern unterschiedlich angelegt, beinhalten aber typischerweise die folgenden Schritte:
- dezidierte Äußerung des Gründungsinteresses und Ausdruck des Willens zu unternehmerischer Selbstständigkeit

- Informierung über die generellen Anforderungen einer Gründung und die speziellen Voraussetzungen einer Förderung mit Einstiegsgeld

- Nachweis persönlicher und fachlicher Voraussetzungen für eine selbstständige Erwerbstätigkeit

- Weiterentwicklung und Konkretisierung des Gründungsprojekts im Businessplan einschließlich der Finanzierungsund Ertragsplanung.

Meist sind die konkreten Verfahrensschritte per ermessenslenkender Weisung oder per legitimierter Arbeitsroutine vorgegeben, lassen aber Handlungsspielraum für ihre Ausgestaltung durch die Integrationsfachkräfte. Deshalb achten viele Jobcenter auf entsprechende wirtschaftsfachliche Voraussetzungen ihrer Fachkräfte. Da Gründungsberatung umfangreiche Kenntnisse voraussetzt, erscheint es wenig effizient, sämtliche Integrationsfachkräfte derartige Kompetenzen erwerben zu lassen. In den meisten der von uns untersuchten Jobcenter wurde stattdessen eine Strategie der Spezialisierung für die Betreuung von Gründungsprozessen gewählt. Diese Prozeduralisierungsroutine liegt im Schnittfeld aller drei Erwartungshorizonte. Sie setzt bei der Initiative der Klienten als Einzelfall an, bestimmt jedoch mit vergleichsweise grober Kategorisierungsarbeit standardisiert darüber, wer zur nächsten Stufe des Vergabeprozesses vorstößt; abhängig vom Grad des Fachwissens der Integrationsfachkräfte kann die Gründungsberatung mit unternehmerischem Wissen angereichert werden.

\subsection{Bindung der Entscheidung an externe Expertise}

Die stufenförmige Abfolge der Verfahrensschritte bietet ein routinisiertes Handlungsschema, das zunächst nur aufseiten der Gründenden wiederholte Entscheidungen zur Fortsetzung des Gründungsvorhabens erfordert (Selbstselektion). Für die Fachkräfte ist die Entscheidungsroutine zur Förderung dagegen gekoppelt an die externe Expertise der fachkundigen Stellen in der letzten Bearbeitungsphase. Die Arbeitshilfe der BA (2010, S. 8f.) setzt als Grundlage die „Prüfung der Tragfähigkeit des Gründungsvorhabens“ voraus und empfiehlt als Entscheidungshilfe die „schriftliche Einschätzung" einer fachkundigen Stelle (z. B. Kammerorganisationen, Gründerzentren, Fachverbände und Kreditinstitute). In der Befragung geben fast alle Integrationsfachkräfte an, dass sie die Befürwortung oder Ablehnung der fachkundigen Stelle unmittelbar in eine entsprechende Förderentscheidung umsetzen. De facto wird damit in den allermeisten Fällen die Entscheidung auf eine fachkundige Stelle ausgelagert, auch wenn sie formal im Jobcenter getroffen wird. Nur wenige Integrationsfachkräfte weichen in Ausnahmefällen von dieser externen Bewertung ab, wenn sie besondere Merkmale des Falles darin nicht hinreichend berücksichtigt finden. 
Im Selektionsprozess, der zur Förderentscheidung führt, verbinden sich die Auswahlmechanismen miteinander: erstens, die Selbstselektion der Gründenden während der Gründungsvorbereitung (in der Bewältigung von Planungsaufgaben und im Nachweis notwendiger Voraussetzungen) und, zweitens, gegen Ende dieses Prozesses das Tragfähigkeitsurteil der fachkundigen Stelle. Die Wirkung beider Mechanismen wird, drittens, dem stufenförmigen Verfahren folgend durch Interventionen der Integrationsfachkräfte beeinflusst, die wiederum eng mit dem Grad der Spezialisierung zusammenhängen. Die Tragfähigkeitsbescheinigung verschafft der Entscheidung externe Legitimation und bedient den Erwartungshorizont der Gründungsberatung. Doch wird die Bewertung der Gründungsprojekte durch Externe auf ein dichotomes Signal reduziert (Tragfähigkeitsbescheinigung liegt vor/ liegt nicht vor), das der Idee der Einzelfallorientierung zuwiderläuft. In dieser reduzierten Kommunikationspraxis werden unternehmerisch-fachliche Aspekte einerseits und persönliche Umstände andererseits getrennt und verschiedenen Akteuren zugewiesen (den fachkundigen Stellen respektive den Integrationsfachkräften), die von der jeweils anderen Seite wenig wissen.

\subsection{Kompetenzaufbau durch Spezialisierung}

Die arbeitsteilige Spezialisierung, die zu einer engeren Abstimmung der Bearbeitungsschritte innerhalb der Jobcenter führt, fördert die Entwicklung von Verfahrensroutinen. Grundsätzlich variiert die Ausgestaltung des Auswahlprozesses und damit seine selektive Wirkung von Jobcenter zu Jobcenter in beträchtlichem Maße. Ein zentraler Unterschied ist, ob und inwieweit die Handhabung des Einstiegsgeldes spezialisierten Mitarbeitern übertragen wird. Einerseits bietet es sich angesichts des Beratungsanspruchs an, die Betreuung von Gründungsanliegen und Selbstständigen bei einigen wenigen Fachkräften zu bündeln. Andererseits sind die potenziellen Förderfälle selbst in den Jobcentern mit vergleichsweise hohen Förderquoten so selten, dass der Ressourcenaufwand einer Spezialisierung unverhältnismäßig hoch erscheinen kann. Schon die Spezialisierung einzelner Mitarbeiter ist ein organisationales Projekt unter den Bedingungen der Ressourcenrestriktion, d.h. sie muss im Vergleich mit alternativen Ressourcenaufwendungen begründet und immer wieder verteidigt werden. Ein wichtiger Effekt ist die Annäherung der Handlungsstrategien der Integrationsfachkräfte in einem spezialisierten Jobcenter. Im Ganzen betrachtet führen verschiedene Spezialisierungsstrategien dazu, dass sich die Unterschiede in der Praxis der Einstiegsgeldvergabe zwischen Jobcentern vergrößern, während sie sich innerhalb dieser eher verringern. Allerdings fehlen zuverlässige Erfolgsindikatoren über die geförderten Gründungen. Die Fachkräfte erhalten kaum systematische Informationen über den Markterfolg und den Verbleib der geförderten Gründungen.
Spezialisierte Fallbearbeitung vervielfältigt die Schnittstellen, die auf dem Weg von der ersten Interessenbekundung bis zur letztendlich gültigen Förderentscheidung überbrückt werden müssen und dies umso mehr, je differenzierter die Spezialisierung ausgeprägt ist. Es muss geklärt werden, zu welchem Zeitpunkt Gründungsinteressierte an die Spezialisten verwiesen werden (z. B. unmittelbar auf die erste Interessenbekundung hin oder erst nach eingehender Informierung und Vorklärung) und wann der Wechsel zurück erfolgt (z. B. schon direkt nach der Gründung oder später). Die Spezialisierung wird häufig als Chance genutzt, das Wissen und Interesse ausgewählter Mitarbeiter gezielt weiterzuentwickeln und damit die Unterstützungsmöglichkeiten zu verbessern. Ferner erleichtert sie die Zusammenarbeit mit den externen Kooperationspartnern der Jobcenter (z. B. Industrie- und Handelskammern oder lokalen Gründungsinitiativen). Damit wird zwar der Austausch mit den fachkundigen Stellen verbessert und weitergehend auf den Erwartungshorizont der Gründungsberatung eingegangen. Doch im Sinne der Einzelfallorientierung bleibt die getrennte Bewertung unternehmerischer und persönlicher Aspekte der Klienten problematisch; in den nicht spezialisierten Jobcentern kommt dieser Differenz wegen der geringeren Beratungstiefe weniger Bedeutung zu.

\subsection{Komplexitätsreduktion durch Verhaltens- und Plausibilitätscheck}

Unabhängig von der Frage der Spezialisierung nutzen alle befragten Fachkräfte über den gesamten Betreuungsprozess hinweg ein Bündel von Kriterien, die sich am normativen Leitbild einer Unternehmerpersönlichkeit orientieren, um eine Einschätzung zu gewinnen, wer zur Gruppe der aussichtsreichen Gründungswilligen zählt und wer nicht. Dieses Leitbild umfasst mehr oder weniger konkrete Persönlichkeitsmerkmale, die in hohem Maße subjektiver Einschätzung unterliegen. Es ist nicht als formal abzuarbeitende Liste verfügbar, sondern als ein kulturelles Deutungsschema, das in ähnlichen Formulierungen auch in den externen Beratungsstellen und von den Jobcenter-Leitungen vorgebracht wird. ${ }^{2}$ Die befragten Integrationsfachkräfte erwarten von den gründungswilligen Personen vor allem Flexibilität und Kommunikationsfähigkeit, Lernbereitschaft und Kreativität, Engagement und (mit besonders häufiger Nennung) Zielstrebigkeit. Inwieweit diese Eigenschaften des „klassischen Unternehmers“ (Bührmann 2012) unterschiedlichsten Formen von Selbstständigkeit angemessen sind, wird nicht hinterfragt.

2 Die Wirkmächtigkeit dieses kulturellen Stereotyps zeigt sich $u$. a. in der in Beratungsliteratur wie Gründungsforschung vorherrschenden Annahme, dass die Schwierigkeiten der Unternehmensgründung eine spezifische Persönlichkeit mit besonderen Veranlagungen erfordern (beispielsweise in der "Starthilfe"-Broschüre zur Existenzgründung des Bundesministeriums für Wirtschaft und Technologie 2010, S. 9ff). 
Die Orientierung an diesem Leitbild dient den Fachkräften als fachlich fundiertes Schema für eine sachlich begründete Urteilsfindung. Für ihre Alltagspraxis machen sie es als Handlungsroutine handhabbar, indem sie eine Reihe von Fragen entwickeln, mit denen sie im Betreuungsgespräch diese Kriterien überprüfen. Damit transferieren sie das Unternehmerleitbild in eine Art impliziter Checkliste, die sie im Gespräch mit praktisch erprobten Formulierungen abarbeiten. Die Gesprächsführung nimmt den Charakter einer fortlaufenden Überprüfung des Gründungsvorhabens anhand einer flexibel anwendbaren Indikatorensammlung an. Beispielsweise fragen die Fachkräfte im Erstgespräch gezielt nach gründungsrelevanten Kompetenzen, wie Fachwissen, beruflichen Erfahrungen und kaufmännischen Kenntnissen; Einsatzbereitschaft und Kundenorientierung der Klienten beurteilen sie anhand der mündlichen Darstellung der Anliegen. Je nach Resultat dieser Inspektion erfolgen die Zuordnung zu einer passenden Stufe im Verfahren und die Veranlassung entsprechender Maßnahmen. Auf diese Weise greifen die organisatorische Ablaufstruktur und die alltägliche Interaktionspraxis unmittelbar ineinander.

Diese immanente Prüfmethodik weist zwei Beurteilungsebenen auf: die Ebene der Verhaltensbeobachtung, mit der auf Eigenschaften und Absichten der Person geschlossen wird, und die Ebene der Plausibilitätskontrolle, welche sich auf die Stimmigkeit der inhaltlichen Angaben richtet. Der Verhaltensscheck gibt Auskunft zur Frage: Mit welcher Art von Klient habe ich es im vorliegenden Fall zu tun? Auf dieser Ebene wird nicht nur darauf geachtet, was Gründungswillige antworten, sondern auch wie sie auf Fragen reagieren. Dem Unternehmerleitbild folgend werden Hinweise gesucht auf Eigeninitiative, Motivation und Zielstrebigkeit. In den Interviews nennen die Fachkräfte folgende Verhaltensweisen als Indizien für eine unternehmerische Veranlagung:

- konkrete Idee präsentieren, Materialien mitbringen, Informationen einholen, Stellen kontaktieren (als Hinweise auf Zielstrebigkeit und Eigeninitiative)

- pünktliches Erscheinen, termingerechte Erledigung von Aufgaben, ordentliche äußere Erscheinung, verbindliches Auftreten (als Signale für Zuverlässigkeit und Kundenorientierung)

- Ideen erklären können, Fragen stellen, auf andere zugehen, Kontakte suchen (als Indizien für Motivation und Kommunikationsfähigkeit).

Auf der inhaltlichen Ebene zielt der Plausibilitätscheck auf die Substanz der Geschäftsidee ab, im Sinne der Frage: Wie realitätsnah erfolgt die Planung für das Gründungsvorhaben? In dieser Hinsicht wird der Standardkontext einer Gründung abgefragt: der Konkretisierungsgrad der Geschäftsidee, Informationen über die Konkurrenzsituation, Einschätzungen der potenziellen Nachfrage, Kenntnisse der Branche, Spielräume für die Preiskalkulation. Je nach Vertrautheit mit der Branche und eigenem Erfahrungsstand gehen die Fachkräfte in sehr unterschiedlichem Detaillie- rungsgrad auf die Projektideen ein. In der Regel aber prüfen sie weniger die konkreten Details, sondern eher die Vollständigkeit und Passgenauigkeit der Einzelinformationen - gewissermaßen die Gestalt der Gründungsidee. Bewertet wird sowohl, ob sich die Gründungsinteressierten mit diesen Aspekten überhaupt näher befasst haben, als auch, ob das Ergebnis dieser Auseinandersetzung realistisch erscheint.

Verhaltens- und Plausibilitätscheck erlauben eine indirekte Form der Entscheidungssteuerung als Verfahrensroutine: Die Frageformulierungen deuten an, wo die Fachkräfte Probleme sehen und welche Konsequenzen sie erwarten, ohne diese explizit ansprechen zu müssen. Soweit sie über Expertenkenntnisse verfügen, können sie gezielt nachfragen, ansonsten haben sie sich oft ein individuelles Repertoire von bewährten Standardfragen zugelegt. Damit wird der Handlungsdruck für potenzielle Gründer erhöht, ohne ihnen die Gestaltungsverantwortung abzunehmen. Analytisch betrachtet oszillieren solche Routinen der Komplexitätsreduktion zwischen dem Erwartungshorizont der Gründungsberatung (der inhaltliche Orientierung bietet) und dem der Standardisierung (mit entsprechenden Kategorisierungsleistungen), ohne die Einzelfallorientierung (in Form individuell und situativ anpassbarer Fragebatterien) gänzlich zu vernachlässigen.

\section{Diskussion}

In diesem Beitrag wurden Fragen der Gründungsförderung der Arbeits- und Sozialverwaltung mit Blick auf die tägliche Arbeitspraxis im Jobcenter verhandelt. Für die Umsetzung der gesetzlichen Vorgaben zum Einstiegsgeld sind umfangreiche Erwartungen hinsichtlich Gründungsberatung, Einzelfallorientierung und Standardisierung institutionalisiert. Gegenüber diesen vielfältigen und oft widersprüchlichen Anforderungen entwickeln sich in den Jobcentern Routinen der Prozeduralisierung der Entscheidung durch Selbstselektion und externe Bewertung, des Kompetenzaufbaus durch interne Spezialisierung sowie der Komplexitätsreduktion durch Verhaltens- und Plausibilitätschecks.

Insbesondere die Prozeduralisierung der Entscheidungsverfahren und die Routinen der Komplexitätsreduktion erleichtern es den Fachkräften, eine Balance zu finden zwischen den verschiedenen Erwartungshorizonten. Sie genügen zwar keiner Erwartung vollends, sie bevorzugen aber auch nicht systematisch eine auf Kosten der anderen. Beispielsweise ermöglicht es die Externalisierung der unternehmerischen Expertise, den Anspruch auf Gründungsberatung und Einzelfallorientierung aufrechtzuerhalten, aber gleichzeitig die Entscheidung an eine formale Voraussetzung zu koppeln. Mit der Spezialisierung und der damit verbundenen Tendenz zur Professionalisierung wird der Erwartungshorizont der Gründungsberatung innerhalb des Jobcenters gestärkt. Diese Stra- 
tegie kommt zudem der Erwartung der Einzelfallorientierung entgegen, sofern ausreichend Zeit für die individuelle Begleitung bleibt, gerät aber in ein Spannungsverhältnis zum Erwartungshorizont der Standardisierung - und auch zum Urteil der fachkundigen Stelle. Denn das Beratungsverständnis und die Begleitungsmöglichkeiten von Jobcenter und externer Gründungsberatung sind unterschiedlich ausgelegt und viele Fachkräfte stehen dem externen Urteil kritisch gegenüber (Pongratz et al. 2013a, S. 98ff.). So haben externe Beratungsstellen selten die Alternativen der Arbeitsmarktintegration für die Gründungswilligen im Blick, die im Jobcenter einen zentralen Entscheidungsmaßstab bilden. Die Sichtweisen interner und externer Spezialisten könnten sich durchaus komplementär ergänzen, doch sind die beiden Verfahrensweisen bislang nicht systematisch aufeinander abgestimmt und der kommunikative Austausch bleibt spärlich.

Dass die Jobcenter nicht allen Erwartungen im Kontext der Gründungsförderung voll entsprechen, ist absehbar und stellt nicht grundsätzlich das Einstiegsgeld als Instrument der aktiven Arbeitsmarktpolitik infrage. Denn bei allen Schwierigkeiten der Gesetzesimplementation kommt der Einstiegsgeldförderung eine strategische Bedeutung als echte Integrationsalternative zu. Das gilt gerade für Gruppen, die üblicherweise größere Schwierigkeiten am Arbeitsmarkt haben. Quantitative Studien zur Wirkung des Einstiegsgeldes zeigen nicht nur vergleichsweise gute Ergebnisse für alle Teilnehmenden (Nivorozhkin/Wolff 2012), sondern auch überdurchschnittliche Effekte bei Migranten und Langzeitarbeitslosen (Wolff et al. 2013). Das Instrument eröffnet den Jobcentern die Möglichkeit, Einfluss auf das Gründungsgeschehen im SGB II zu nehmen und so den Bezug von Unterstützungsleistungen nach einer Gründung zu reduzieren. Die Geförderten wiederum messen dem Einstiegsgeld trotz der geringen Förderhöhe und der größtenteils nur wenige Monate langen Förderdauer zumeist eine große Bedeutung zu (ebd.). Dies erklärt sich auch daraus, dass gerade in den Anfangsmonaten einer Selbstständigkeit Aufgaben anstehen, die Leistungsberechtigte des SGB II ohne Hilfe finanziell nur schwer stemmen können - vom Gang zum Steuerberater bis zu gestiegenen Mobilitätskosten.

Die geschilderten Verfahrensroutinen ermöglichen es vielen der befragten Fachkräfte, einen sicheren Umgang mit den Anforderungen der Einstiegsgeldvergabe zu entwickeln. Sie berichten aber auch von Kollegen, die den Einsatz des Förderinstruments nach Möglichkeit vermeiden (Pongratz et al. 2013a, S. 75); diesen bietet eine Spezialisierung im Jobcenter die Gelegenheit, das ungeliebte Thema abzugeben. Trotz aller Routinisierung bleiben erhebliche Divergenzen in der Förderungspraxis bestehen, die dazu führen, dass ähnliche Gründungsanliegen recht unterschiedliche Förderungschancen haben - sowohl zwischen den Jobcentern als auch bei verschiedenen Fachkräften innerhalb eines Jobcenters. Die Spezialisierung bietet den Vorteil, dass sie die Auseinandersetzung zum Thema Gründungsförderung intensiviert und so zur Klärung der internen Verfahrensweisen beiträgt. Demgegenüber bleibt der Informationsaustausch der Jobcenter untereinander zu ihren Erfahrungen mit dem Einstiegsgeld ebenso beschränkt wie zwischen Jobcentern und externen Gründungsberatungen.

Für die gründungswilligen Klienten schaffen die Verfahrensroutinen einen Rahmen, der die Transparenz des Bearbeitungsverfahrens erhöht und sie immer wieder vor Entscheidungssituationen stellt, die ihren Klärungsprozess vorantreiben. Wie die Fallstudien der Beratungsinteraktionen zeigen, die wir an anderer Stelle intensiv ausgewertet haben (Pongratz et al. 2013b), kommen die Klienten damit in unterschiedlicher Weise zurecht. Einige können die Verfahren nutzen, um ihr Gründungsprojekt weiterzuentwickeln und zu konkretisieren, andere sehen sich durch starre Vorgaben in ihrem Vorhaben eher gehindert - und versuchen, diese zu umgehen. Bei Letzteren kommt es auch bei der Einstiegsgeldvergabe zu jenen Disziplinierungseffekten, die in der reformierten Arbeits- und Sozialpolitik generell beobachtet werden (Ludwig-Mayerhofer et al. 2008). Insgesamt betrachtet, werten wir die Entwicklung der Verfahrensroutinen als einen Fortschritt, der zur Handlungssicherheit der Fachkräfte und zur Transparenz der Verfahren beiträgt. Angesichts der verbleibenden Diskrepanzen und Widersprüche aber bleibt die Frage einer angemessenen Begleitung von Gründungsprozessen im Rahmen der Sozial- und Arbeitsverwaltung eine anhaltende Herausforderung. Eine Politik, die sich auf die Reduzierung von Förderzahlen beschränkt, löst weder die Entscheidungsprobleme der Fachkräfte noch hilft sie den Klienten bei der Klärung der Erfolgsaussichten einer Existenzgründung.

\section{LITERATUR}

Bernhard, S. (2012a): Der Gründungszuschuss vor und nach der Reform, in: Sozialer Fortschritt 61 (8), S. 182-190

Bernhard, S. (2012b): Selbständige in der Arbeitsverwaltung. Eine Rahmenanalyse nach Goffman, in: Bereswill, M./Figlestahler, C./Haller, L. Y./Perels, M./ Zahradnik, F. (Hrsg.): Wechselverhältnisse im Wohlfahrtstaat. Dynamiken gesellschaftlicher Justierungsprozesse, Münster, S. 234-255

Bernhard, S./Pongratz, H. J./Wolff, J. (2013): Das Einstiegsgeld: Gedämpfte Erwartungen in den Jobcentern, IAB Kurzbericht 27/2013, Nürnberg

Bührmann, A. D. (2012): Unternehmertum jenseits des Normalunternehmertums: Für eine praxistheoretisch inspirierte Erforschung unternehmerischer Aktivitäten, in: Berliner Journal für Soziologie 22 (1), S. 129-156

Bundesagentur für Arbeit (BA) (2009): Einführung einer zukunftsorientierten BA-Beratungskonzeption ab 2009, HEGA 05/09-06

Bundesagentur für Arbeit (BA) (2010): SGB II Arbeitshilfe: Einstiegsgeld nach § 16b SGB II, Stand: März

Bundesagentur für Arbeit (BA) (2013): Einführung der Beratungskonzeption im Rechtskreis SGB II (JC), HEGA 08/2013-7b

Bundesministerium für Wirtschaft und Technologie (2010): Starthilfe. Der erfolgreiche Weg in die Selbstständigkeit, Berlin

Caliendo, M./Kritikos, A. (2007): Die reformierte Gründungsforschung für Arbeitslose: Chancen und Risiken, IZA Discussion Paper (3114), Bonn

Caliendo, M./Künn, S./Wießner, F. (2009): Erfolgsgeschichte mit zu frühem

Ende. Ich-AG und Überbrückungsgeld: Institut für Arbeitsmarkt- und Berufsforschung, IAB-Kurzbericht 3/2009, Nürnberg

Deutscher Bundestag (2003): Entwurf eines Vierten Gesetzes für moderne Dienstleistungen am Arbeitsmarkt, Drucksache 15/1516 vom 05.09.

Dunkel, W. (2011): Arbeit in sozialen Dienstleistungsorganisationen: die Interaktion mit dem Klienten, in: Evers, A./Heinze, R. G./Olk, T. (Hrsg.): Handbuch Soziale Dienste, Wiesbaden, S. 187-205

Flick, U. (2004): Triangulation. Eine Einführung, Wiesbaden

Hielscher, V./Ochs, P. (2012): Das prekäre Dienstleistungsversprechen der öffentlichen Arbeitsverwaltung, in: Bothfeld, S./Sesselmeier, W./Bogedan, C. (Hrsg.): Arbeitsmarktpolitik in der sozialen Marktwirtschaft. Vom Arbeitsförderungsgesetz zum Sozialgesetzbuch II und III, Wiesbaden, S. 249-259

Hirschauer, S. (2001): Ethnographisches Schreiben und die Schweigsamkeit des Sozialen. Zur Methodologie der Beschreibung, in: Zeitschrift für Soziologie 30 (6), S. 429-451

Ludwig-Mayerhofer, W./Behrend, O./Sondermann, A. (2008): Disziplinieren und Motivieren: Zur Praxis der neuen Arbeitsmarktpolitik, in: Evers, A./Heinze, R. G. (Hrsg.): Sozialpolitik. Ökonomisierung und Entgrenzung, Wiesbaden, S. $276-300$

Metzger, G./Ullrich, K. (2013): Gründungsgeschehen auf dem Tiefpunkt kein Anstieg in Sicht. KfW Gründungsmonitor, in: KfW Economic Research, https://www.kfw.de/Presse-Newsroom/Pressetermine/Dokumente-2013/ Gr\%C3 \%BCndungsmonitor-2013/Bericht-Gr\%C3 \%BCndungsmonitor-2013.pdf. Nivorozhkin, A./Wolff, J. (2012): Start me up:The effectiveness of a self-employment programme for needy unemployed people in Germany, in: Journal of Small Business and Entrepreneurship 25 (4), S. 499-518 
Pongratz, H. J./Bernhard, S./Abbenhardt, L. (2013a): Fiktion und Substanz. Unsicherheitsbearbeitung im Gründungsprozess, München/Nürnberg (Manuskript)

Pongratz, H. J./Bernhard, S./Wolff, J./Promberger, M. (2013b): Selbständig statt leistungsberechtigt. Eine Implementationsstudie zur Handhabung des Einstiegsgeldes in den Jobcentern, IAB Forschungsbericht 3/2013, Nürnberg Rüb, F./Werner, D. (2007): Typisierung von SGB II Trägern, IAB Forschungsbericht $1 / 2007$, Nürnberg

Schütz, H. (2009): Neue und alte Regelsteuerung in der deutschen Arbeitsverwaltung, in: Bothfeld, S./Sesselmeier, W./Bogedan, C. (Hrsg.): Arbeitsmarktpolitik in der sozialen Marktwirtschaft, Wiesbaden, S. 163-177

Sternberg, R./Vorderwülbecke, A./Brixy, U. (2013): Global Entrepreneurship Monitor. Unternehmensgründungen im weltweiten Vergleich. Länderbericht Deutschland 2012, Global Entrepreneurship Research Association (GERA)

Hannover/Nürnberg

Witzel, A./Reiter, H. (2012): The problem-centred interview, London

Wolff, J./Nivorozhkin, A./Bernhard, S. (2014): You can go your own way! The longterm effectiveness of a self-employment programme for German welfare recipients, Manuskript

\section{AUTOREN}

STEFAN BERNHARD, Dr. rer. pol., Wissenschaftlicher Mitarbeiter am Institut für Arbeitsmarkt- und Berufsforschung (IAB) in Nürnberg. Arbeitsschwerpunkte: Arbeitsmarkt- und Sozialpolitik, Europasoziologie, Netzwerkanalyse, Politische Soziologie, Qualitative Sozialforschung und Wirtschaftssoziologie.

@ stefan.bernhard@iab.de

HANS J. PONGRATZ, Prof. Dr., Institut für Sozialwissenschaftliche Forschung (ISF) München und Ludwig-Maximilians-Universität (LMU) München. Arbeitsschwerpunkte: Arbeits- und Organisationssoziologie, Arbeitsmarkt und berufliche Selbstständigkeit, Wandel in Betrieben und Gesellschaft, qualitative Sozialforschung.

@ hans.pongratz@isf-muenchen.de 